\title{
INTERNALIZAÇÃO DE FARMOQUÍMICOS \& MEDICAMENTOS PARA DOENÇAS TROPICAIS NEGLIGENCIADAS: PROPOSTA DE INTERAÇÃO ENTRE GOVERNO - UNIVERSIDADE - EMPRESA
}

\author{
Jorge Lima de Magalhães* e Núbia Boechat \\ Departamento de Síntese em Fármacos, Área Gestão da Inovação Tecnológica, Fundação Oswaldo Cruz/Farmanguinhos, Rua \\ Sizenando Nabuco, 100, 21041-250 Rio de Janeiro - RJ, Brasil \\ Adelaide Maria de Souza Antunes \\ Instituto Nacional de Propriedade Intelectual, Praça Mauá, 7, 20081-240 Rio de Janeiro - RJ / Escola de Química, Universidade \\ Federal do Rio de Janeiro, 21949-900 Rio de Janeiro - RJ, Brasil
}

Recebido em 10/5/11; aceito em 16/8/11; publicado na web em 26/9/11

\begin{abstract}
INTERNALIZING ACTIVE PHARMACEUTICAL INGREDIENTS \& MEDICINES FOR NEGLECTED TROPICAL DISEASES: PROPOSAL FOR INTERACTION AMONG GOVERNMENT - UNIVERSITY - INDUSTRY. History shows that public health actions to combat epidemics are most effective when there is constant synergy of both government and society, exemplified by prevention actions in the fight against dengue fever, H1N1 influenza etc. Private-public-partnerships (PPP) can also provide more dynamic and consistent results in production through interaction among Government, University and Enterprise actors. This article puts forward proposals to tackle the problem of neglected tropical diseases via the formation of a Management Council using Etzkowitz's Triple Helix concept to attenuate this public health issue in Brazil.
\end{abstract}

Keywords: triple helix; active pharmaceutical ingredient; neglected tropical disease.

\section{INTRODUÇÃO}

O sistema nacional de inovação (SNI) de um país pode ser definido como o conjunto de instituições (públicas, privadas, produtivas, culturais, econômicas, científicas ou tecnológicas) e de suas respectivas interações que proporcionam o ambiente de inovação e desenvolvimento de uma nação. Segundo a literatura sobre economia da tecnologia, a presença de um SNI induz a transformação do conhecimento, a tecnologia em desenvolvimento e o crescimento econômico, oferecendo as condições para que um país abandone condições de pobreza e exclusão social. ${ }^{1}$

O Brasil pertence ao grupo de países que possuem SNI imaturos, aqueles em que ainda se observa a presença de algumas instituições com interações frágeis entre seus participantes (SNI maduros são os países líderes em desenvolvimento e tecnologia, como EUA e Japão; já os países em processos de catching up são os que possuem boas instituições que interagem entre si, mas estão em um nível abaixo dos países líderes, como Taiwan e Coreia; e nos sistemas imaturos estão Brasil, Índia, México). Segundo a economia da tecnologia, um dos fatores é a necessidade da incorporação da ciência nos negócios de acordo com as características de cada setor, pois estes apresentam trajetórias tecnológicas diferentes. ${ }^{2}$

Desta forma, é preciso um melhor manejo do conhecimento e da tecnologia, principalmente com a adaptação às condições reais de cada local. Para isso, é preciso interligar todos os setores da sociedade, desde instituições de pesquisas e universidades, o campo industrial, a saúde pública e a atenção médica, pois ainda há carência de ações intersetoriais visando garantir a sustentabilidade de ações com outros setores. Contudo, a cooperação tecnológica, seja por meio de entes governamentais e/ou privados, e até as Organizações Não Governamentais (ONGs), tem fortalecido gradativamente o processo de desenvolvimento.

Cabe registrar importantes avanços ocorridos nos últimos anos através de redes de cooperação promovidas por organizações como

\footnotetext{
*e-mail: jorgelim@far.fiocruz.br
}

TB Alliance (Global Alliance for TB Drug Development) ou Drug for Neglected Disease Iniciative (DNDi). Estas iniciativas visam parcerias com o setor público ou privado para a pesquisa de novos produtos no combate às doenças negligenciadas. ${ }^{3}$

Buse e Walt ${ }^{4}$ ressaltam como exemplo de mecanismo adotado para promover o desenvolvimento específico de novos medicamentos, vacinas e métodos diagnósticos para uso em doenças tropicais negligenciadas (DTN) as PPP, cuja parceria para o desenvolvimento de produto (PDP) confere união de forças para obtenção de um resultado. Pode-se melhor exemplificar com a obtenção da dose fixa combinada para malária de artesunato + mefloquina. Este projeto foi lançado em 2007 através da parceria iniciada em 2002 pela iniciativa de medicamentos para doenças negligenciadas - o DND $i$ (fundada em 2003 pelos Médicos Sem Fronteiras para atuar em prol do interesse público preenchendo as lacunas de Pesquisa e Desenvolvimento (P\&D) para algumas DTN), o laboratório oficial Farmanguinhos e a empresa farmacêutica Sanofi-Aventis.

Esses mecanismos de cooperação representam um avanço substancial e contribuem para atuar na problemática que representam as DTN, embora apresentem algumas limitações, como ter o foco no escopo de trabalho em P\&D e, desta forma, direcionar menos atenção às questões de acesso aos produtos pelas populações carentes e os respectivos governos. Também há dificuldades neste modelo, quando se considera que foi concebido por uma ação dos 'países do norte' para desenvolver soluções dos 'países do sul' - visão ultrapassada do tempo colonial, da medicina tropical. ${ }^{5}$ No caso brasileiro, onde a compra dos medicamentos para DTN é garantida pelo governo, há que se garantir políticas para distribuição até a população necessitada.

Vieira e Albuquerque ${ }^{6}$ descreveram que as alianças estratégicas entre as empresas e PPP envolvem não somente o governo, mas também as universidades, centros de pesquisa e empresas - todas são necessárias à continuidade do processo inovador. Em outras palavras, à medida que o processo de inovação se torna mais complexo e as empresas se tornam mais especializadas, a geração de inovações passa a depender crescentemente de "redes cooperativas" e de arranjos institucionais de apoio. 
Nesse sentido, para a gestão do conhecimento, se estabelecem linhas divisórias claras entre os que estão capacitados a promover ou a participar ativamente em uma dinâmica de inovação e aprendizado e aqueles que foram, ou tendem a ser, deslocados e marginalizados pelas transformações na base técnico-produtiva. No aspecto econômico, os que se mantêm mais dinâmicos e competitivos são os segmentos e organizações que se colocam à frente do processo inovador.

Os acordos para desenvolvimento de produção constituem boa ferramenta, porém devem ser estabelecidos de forma integrada com todos os atores a fim de maximizar e convergir em todas as ações. No campo da saúde pública, é importante o envolvimento dos laboratórios farmacêuticos oficiais (LFO), que constituem verdadeiro patrimônio público e são estratégicos para o Governo no estabelecimento de políticas e na produção de medicamentos, principalmente para atenção básica (produtos necessários às ações e procedimentos compreendidos na atenção básica de saúde - analgésicos, antitérmicos etc.) e a síndrome da imunodeficiência adquirida (AIDS), cujo potencial de capacidade produtiva está em 16,6 bilhões de unidades farmacêuticas (UFs). ${ }^{8-10}$

Mesmo diante dos avanços concebidos por estas parcerias, a $\mathrm{P}$, D \& I nos países em desenvolvimento ainda é pífia se comparada com a base tecnológica e produtiva das nações desenvolvidas, como EUA. ${ }^{11}$ Segundo Gadelha (2008), há que se buscar um enfoque mais dinâmico na economia, alternativo ao paradigma neoclássico dominante (remonta ao processo de impulso expansivo do capitalismo mediante, ${ }^{12}$ respectivamente, o processo de "desenvolvimento das forças produtivas", da "destruição criativa" de corte schumpeteriano, donde resulta em oportunidades e desafios para o desenvolvimento do país), em particular na análise do complexo econômico industrial da saúde (CEIS) no Brasil. Tendo em vista no campo da saúde haver uma deterioração do potencial de inovação e uma vulnerabilidade na política externa, causando uma dicotomia na relação entre o sistema de saúde e o sistema econômico industrial. ${ }^{13}$

No âmbito do Estado brasileiro, existem diversas instituições envolvidas no setor de medicamentos, desde ministérios subordinados à Presidência da República até agências independentes e subordinadas a outros poderes. Este aparato estatal encerra um tratamento fragmentário do sistema de saúde brasileiro já que, em alguns casos, as entidades e agências possuem posições e objetivos antagônicos e se encontram divididas em campos distintos, nem sempre absolutamente contraditórios. ${ }^{14}$

Grandes diferenças permeiam as diversas vantagens comerciais, políticas e acadêmicas como é observado no Quadro 1. Portanto, ainda é um grande desafio conscientizar as autoridades sobre a necessidade do equilíbrio de interesses para a conquista de uma sociedade mais justa e equânime no que diz respeito a acesso à informação, aos medicamentos e tratamentos à sua população. Tendo em vista que as três esferas precisam conviver, uma nova cultura de balanceamento entre as diferenças existentes deve ser fomentada, como a cultura de inovação, o tempo-qualidade (instantes vivenciados em sintonia passam rapidamente $;{ }^{15}$ os caracterizados pelo tédio decorrem devagar; horas podem ser vivenciadas como minutos e, inversamente, minutos como horas; o passado pode estender-se até o futuro, ou este, até o presente) e o pensar global com atitudes para o desenvolvimento local (glocalização). ${ }^{16}$

Estas disparidades podem ser transportas para o campo das DTN e uma das formas de minimizar estas defasagens em prol do benefício da sociedade é utilizar a abordagem da hélice tríplice (HT).

A HT propõe um modelo espiral de inovação baseado na geração e distribuição do conhecimento. É um mecanismo complexo e dinâmico de interação contínua entre três hélices, Governo-Universidade-Empresa (G-U-E) numa espiral infinita de transições, onde se possam implantar políticas consistentes em rede para melhoria comum, no que se refere à geração de conhecimentos, inovação e desenvolvimento econômico.

A estrutura é centrada em objetivos comuns das três esferas como um indicador, porém multidisciplinares, em que todos envolvidos se comuniquem de forma dinâmica, complementando estudos, cedendo conhecimentos, avançando na ciência e, assim, socializando informações para tomada de decisão e o avanço da economia regional. ${ }^{17,18}$

\section{ABORDAGEM DA HÉLICE TRÍPLICE E A RELAÇÃO NO ENFRENTAMENTO DAS DOENÇAS TROPICAIS NEGLIGENCIADAS}

Considera-se que no século XXI, a fronteira entre a ciência e tecnologia, a política, o social e as instituições jurídicas continuarão a influenciar e depender uns dos outros. Nesse sentido, a convergência entre cientistas, empresas e parlamentares é premente, pois precisam compreender as implicações sociais e jurídicas de seus trabalhos antes de seguir adiante, tendo em vista serem constantemente desafiados por rápidos avanços na tecnologia. Assim, o uso do conceito HT ajuda reduzir o fosso entre ciência, sociedade e leis governamentais. ${ }^{19}$

O panorama das DTN, segundo a Organização Mundial da Saúde (OMS), não dispõe de tratamentos eficazes ou adequados, e são: úlcera de Buruli (infecção por Mycobacterium ulcerans), doença de Chagas, cisticercose, dengue, dracunculose (verme de Guiné), fascioliose, treponematoses endêmicas, infecções transmitidas por alimentos trematódeos, tripanossomíase humana africana (doença do sono), leishmaniose, hanseníase, filariose linfática (elefantíase), oncocercose (cegueira dos rios), raiva, esquistossomose, tracoma e helmintíases transmitidas através do solo. Estima-se que há cerca de 1 bilhão de pessoas (1/6 da população mundial) sofrendo de alguma doença negligenciada. Embora sejam típicas de países pobres e atinjam primordialmente as populações dos países em desenvolvimento, elas têm aumentado nos países desenvolvidos, gerando um impacto devastador sobre a humanidade..$^{20} \mathrm{O}$ Estado brasileiro importou entre 2005 e 2009 cerca de US\$ 760 milhões de fármacos para produzir medicamentos para estas doenças. Já em medicamentos para DTN foram gastos R $\$ 145$ milhões no mesmo período. ${ }^{10,21,22}$

Diante da complexidade que o tema de saúde pública abrange e do emblemático poderio do setor farmacêutico, que movimenta mais de US\$ 800 bilhões/ano (IMS Health, 2010), é que a abordagem da HT auxilia na complexidade em tratar as questões brasileiras inerentes às DTN de forma organizada, coesa e produtiva para o país. ${ }^{23}$

Quadro 1. Exemplo de diferenças e fatores existentes no âmbito do governo, universidade e empresa

\begin{tabular}{|lcccc|}
\hline & $\begin{array}{c}\text { Negócios } \\
\text { (empresa) }\end{array}$ & Governo & $\begin{array}{c}\text { Pesquisa \& Desenvolvimento } \\
\text { (universidade) }\end{array}$ & $\begin{array}{c}\text { Equilíbrio } \\
\text { (convívio das 3 esferas) }\end{array}$ \\
\hline Cultura & Econômica & Política & Científica & Inovativa \\
Tempo/Perspectiva & 4 meses & 4 anos & $6-10$ anos & Consciência do tempo-qualidade \\
Perspectiva geográfica & Global & Regional/local & Global & Glocalização \\
Prêmios & Lucro & Reeleição & Conhecimento científico & Desenvolvimento \\
\hline
\end{tabular}

Fonte: Adaptado Seminário Hélice Tríplice na América Latina, Porto Alegre - Nov, 2009 
A teoria de Schumpeter ${ }^{12}$ de destruição criativa mostra como desapareceram os ultrapassados regimes econômicos, mas a HT delineia como os novos regimes irão aparecer. A estruturação desse novo modelo passa por elencar um conjunto de elementos, relações e funções e, em seguida, discutir as atividades específicas e formatos do conhecimento, inovação e ambientes de consenso, além de observar a dinâmica não linear das transições possíveis entre eles. ${ }^{24}$

Para o tema DTN, que não desperta grande interesse das empresas privadas, a não ser o caráter socioambiental e sua respectiva imagem, é que o indutor principal para "girar" a primeira hélice na abordagem HT deverá ser o Estado, com o planejamento e coordenação das ações.

As contribuições da comunidade científica poderiam compor a $2^{\mathrm{a}}$ hélice e as capacidades do empresariado nacional são consideradas posteriormente na $3^{\mathrm{a}}$ hélice. Embora as hélices sejam "giradas" uma após outra, em um ponto de vista teórico, os movimentos podem se sobrepor ou até "girar" simultaneamente em busca de sinergia e ações concretas para obtenção de resultados práticos objetivando um sistema de inovação em DTN (Figura 1).

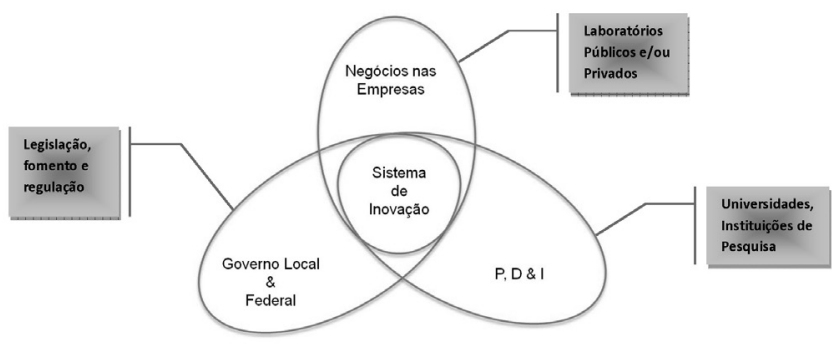

Figura 1. Exemplificação do conceito de hélice tríplice para DTN. Adaptada do seminário HT na América Latina, Porto Alegre (Nov, 2009)

Observa-se que as 3 hélices têm um ponto em comum (inovação) e que este pode aumentar ou diminuir dependendo das interações e necessidades, bem como da velocidade em que uma "gira" em relação à outra.

A implicação política desse equilíbrio obtido, entre as abordagens internas e externas de cada organização, tem em vista o bem comum para a relação ganho-ganha, onde todos lucrem e a sociedade usufrua dos benefícios. A intervenção governamental é essencial, por um lado, incentivando o desenvolvimento das universidades empreendedoras (capacidade organizacional para assumir papéis de liderança em suas áreas de atuação) e, por outro, definindo as regras do jogo, por exemplo, a estruturação da propriedade intelectual, regulamentação e, se for o caso, os sistemas fiscais para fomentar novas empresas. Da mesma forma com as empresas, extraindo o que cada um faz de melhor na sua essência. ${ }^{24}$

Segundo Etzkowitz e Ranga, ${ }^{25}$ a configuração do G-U-E ainda pressupõe a existência de um espaço HT. Este ambiente é norteado por 3 regras básicas: conhecimento, inovação e espaço de consenso, que serão os caminhos para romper as fronteiras de interação entre as esferas institucionais. Principalmente a mudança de paradigma de um modelo laissez-faire (regime de esferas independentes) para um modelo balanceado (regime de esferas que se relacionam), cujo objetivo central é obter um sistema de desenvolvimento constante. Os espaços devem:

- disponibilizar uma estrutura de comunicação com reflexões interativas;

- possibilitar uma estrutura institucional: interseção das esferas considerando a "inovação sobre a inovação" como atores individuais e organizacionais - um assumindo o lugar do outro. Permite criar novos espaços de interação e, se necessário, inventar novos formatos organizacionais. Através deste processo criativo, as relações entre as esferas são continuamente reformuladas em "uma transição sem fim" para aumentar inovação;

- a geração do conhecimento, a difusão e a utilização surgem como resultado de dois processos de comunicação e diferenciação: a) um funcional, entre a ciência e os mercados e, b) um institucional, entre o controle público e privado no nível das universidades, indústrias e governo, que permite vários graus de ajuste seletivo mútuo.

Nesse espaço HT, cabe destacar alguns componentes que permeiam o modelo:

- universidade ou instituições de P\&D: as funções tradicionais da universidade (ensino, pesquisa e extensão) começam a adquirir uma nova função, que é a participação no desenvolvimento socioeconômico - uma "terceira ou quarta missão". Desta forma, amplia-se a capacidade das universidades para gerar tecnologia e, aproveita-se o potencial dos alunos para proporcionar novas ideias e talentos empreendedores;

- instituições híbridas: agrupam-se elementos da academia, indústria e do governo na instância de planejamento/ou suporte por um espaço de tempo ou não, como formação de centros de pesquisa interdisciplinares, consórcios de investigação na indústria-universidade, institutos de investigação, escritórios de transferência de tecnologia nas universidades, empresas e laboratórios de pesquisa do governo, instituições de apoio às empresas (parques de ciência, negócio/incubadoras tecnológicas); instituições financeiras de apoio às novas empresas de base tecnológica (empresas públicas e privadas de capital de risco, redes etc.).

Ao mesmo tempo, a diferenciação interna dentro de cada esfera institucional gera novos tipos de laços e estruturas entre os campos da hélice, tais como serviços de ligação industrial em universidades ou alianças estratégicas entre empresas e a criação de novos mecanismos de integração em rede.

Devido às peculiaridades das instituições, elas exercem influência no momento de defender seus interesses em termos de resultados -é uma questão-chave. Porém, não é mais possível entender as organizações com relação um-para-um. São melhores as sinergias entre os diferentes ambientes de atuação, podendo gerar resultados e reformas institucionais bem melhores e maiores para todos. ${ }^{24}$

Nesta proposta, os atores estarão atuando como indutores em todas as funções, mesmo que esta não seja sua atividade principal. Portanto, a definição dos espaços é diretamente função do sistema HT, que se divide em sub-funções similares ou atividades de sistemas de inovação. Essas tarefas, descritas por Markard e Truffer, ${ }^{26}$ devem permear toda discussão entre o modelo (neo) institucional, como os fatores que influenciam o desenvolvimento, a difusão e utilização de inovações.

Uma condição essencial para a criação e consolidação desse espaço de conhecimento é a existência de uma "massa crítica" de pesquisa acadêmica, bem como as competências específicas de cada instituição na sua área local. ${ }^{27}$ Por isso, universidades e outras instituições produtoras de conhecimento da indústria e das esferas de governo são fundamentais para estabelecer um efetivo conhecimento do espaço. Sinteticamente, é a capacidade integradora da universidade, proporcionando um local neutro para os agentes de outras competências colaborarem, assim como seu fluxo através dos recursos humanos, que cada vez mais se torna o elemento-chave no espaço da cooperação do conhecimento. ${ }^{24}$

Considerando as DTN, o consenso neste espaço da HT deve ser adaptado e, portanto, recomenda-se gerar e manter:

- independências entre as organizações;

- cobertura mais ampla do conceito de governança, incluindo o governo, bem como os atores não governamentais;

- alteração dos limites do Estado, com a mudança e mais transparência dos limites entre os setores público, privado e até voluntários; - $\quad$ interação permanente entre os membros da rede, em função da 
necessidade de intercâmbio de recursos para fins comuns; relação de confiança regulada pelas regras negociadas do jogo e aprovadas pelos participantes;

nível de autonomia do Estado;

o Estado ocupa uma posição de soberania nas parcerias e pode, indiretamente, orientá-las.

Baseado no princípio norteador do modelo HT e da necessidade de um enfrentamento mais abrangente das DTN, propõe-se a coordenação de todas as atividades elencadas no espaço HT, por um Conselho Gestor de Combate às Doenças Negligenciadas (CGC-DTN), cujo objetivo maior seja a soberania nacional em farmoquímicos \& medicamentos para DTN aliada ao fortalecimento da ciência e das empresas nacionais.

\section{INTERNALIZAÇÃO DE FARMOQUÍMICOS \& MEDICAMENTOS PROMOVIDA POR UM CONSELHO GESTOR DE COMBATE ÀS DOENÇAS TROPICAIS NEGLIGENCIADAS (CGC-DTN)}

Atendendo à proposta de aplicação deste trabalho, o CGC-DTN representaria o Estado brasileiro, no âmbito do MS, mas com representantes tanto do poder público como da sociedade civil (Figura 2).
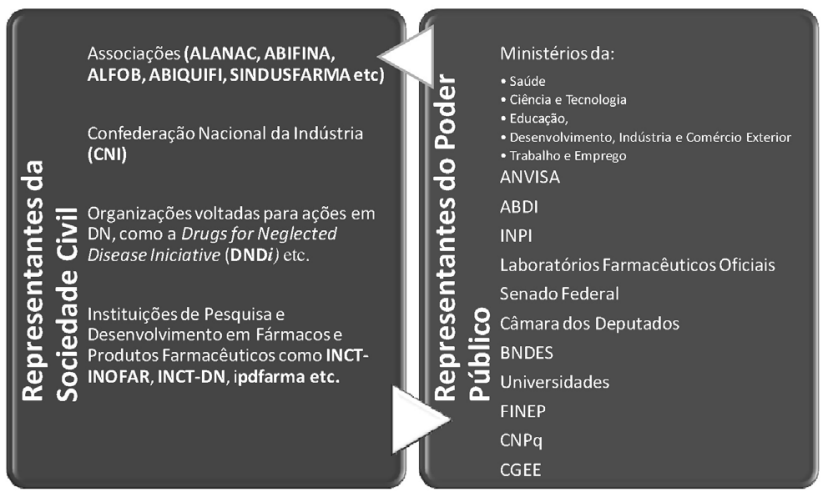

Figura 2. Composição do Conselho Gestor de Combate às DTN

A composição do Conselho ficaria sob o comando do MS objetivando melhor articulação entre todos os atores e, ainda, por ser a área que trata diretamente da atenção à saúde da população brasileira. Seguem exemplos, de alguns ministérios e instituições que comporiam o CGC-DTN:

associações: de laboratórios e empresas como, por exemplo, Associação Laboratórios Farmacêuticos Nacionais (ALANAC), Associação Brasileira das Indústrias de Química Fina, Biotecnologia e suas Especialidades (ABIFINA), Associação dos Laboratórios Farmacêuticos Oficiais do Brasil (ALFOB), Associação Brasileira da Indústria Farmoquímica e Insumos Farmacêuticos (ABIQUIFI), Sindicato das Indústrias Farmacêuticas (SINDUSFARMA) etc;

organizações voltadas para iniciativas em doenças negligenciadas poderão contribuir sensivelmente para o avanço das ações no Conselho como, por exemplo, DND $i$;

- Instituto Nacional de Propriedade Intelectual (INPI): visa auxiliar nas tomadas de decisão e articulações inerentes à propriedade intelectual e às estatísticas de patentes e marcas;

- Ministério da Educação (MEC), Ministério de Desenvolvimento, Indústria e Comércio (MDIC), Ministério de Ciência e Tecnologia (MCT), Ministério de Trabalho e Emprego (MTE) e Ministério da Justiça (MJ) - objetivariam a melhor articulação entre os atores envolvidos de alguma forma com a saúde pública brasileira, no que tange à disponibilização de cientistas e grupos de pesquisa e extensão; fomento da pesquisa e desenvolvimento tecnológico, inclusão e monitoramento dos trabalhadores; procedimentos legais e estruturantes para regulamentações nesta área etc;

- Laboratórios Farmacêuticos Oficiais (LFO): um bom exemplo é a FIOCRUZ, por ser a instituição pública que possui na sua estrutura grande parte da cadeia farmacêutica, perpassando pela pesquisa, desenvolvimento, inovação, ensino e produção de medicamentos e vacinas. Desta forma, podendo contribuir não somente com seus cientistas e grupos de pesquisa, como no desenvolvimento tecnológico de produtos e a própria produção; Agência Nacional de Vigilância Sanitária (ANVISA): que, por ser uma agência reguladora e de vigilância, poderá auxiliar nas decisões, agilizando os caminhos e procedimentos para obtenção de registros, aprimoramento de legislação sanitária pertinente, garantindo a qualidade dos produtos etc;

Câmara dos Deputados e Senado Federal: em razão das formulações de políticas na área em questão. Necessidade de munir os governantes de subsídios, tal como indicadores, a fim de facilitar as tomadas de decisão no Legislativo, visando fortalecer a defesa nacional (associada à defesa nacional em fármacos e medicamentos - capacidade do país ser soberano em determinada área/produto em relação a outras nações) em fármacos \& medicamentos para benefício da sociedade brasileira.

O CGC-DTN, estando estabelecido, deveria promover:

A articulação entre a Política Nacional de Assistência

Farmacêutica e a Política Nacional de Medicamentos com

a Política Nacional de Ciência, Tecnologia e Inovação em

Saúde - PNCTI/S, deve proporcionar por intermédio de ações convergentes o apoio à competitividade, ao estímulo à $P \& D$ nas empresas e universidades com investimento em infraestrutura e, ao incentivo às parcerias, com papel destacado no desenvolvimento do CEIS. ${ }^{28,29}$

Alinhada nessas premissas, uma estrutura do CGC-DTN que, além de articular com os atores da HT, mantenha um canal de comunicação e transparência com a sociedade, expressando a missão, os valores, a visão e estratégia (Figura 3).

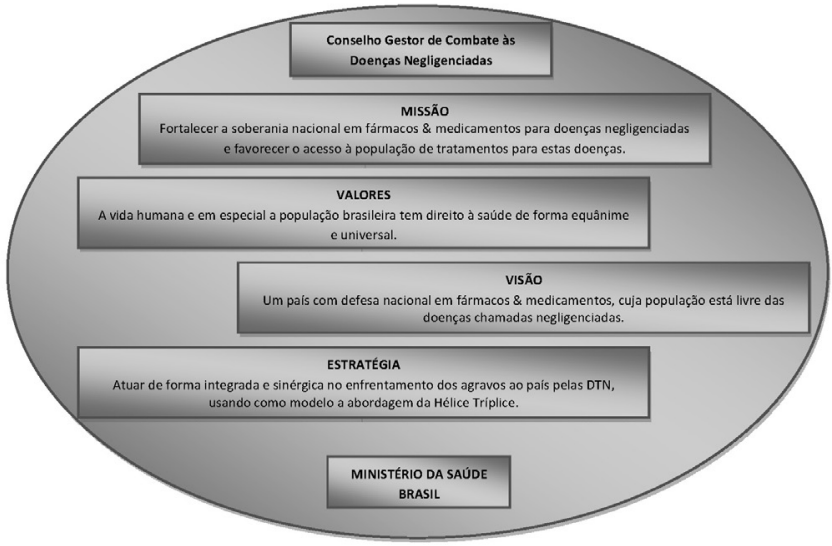

Figura 3. Estrutura do CGC-DTN

Os projetos de atuação do CGC-DTN, se criado, seguiriam os seguintes pressupostos:

$1^{\circ}$ conhecimento da evolução histórica e epidemiológica da saúde pública;

$2^{\circ}$ entendimento da evolução da RENAME e o portfólio dos Laboratórios Farmacêuticos Oficiais;

$3^{\circ}$ ciência da dinâmica do mercado farmacêutico e a comercialização dos medicamentos para as DTN em solo brasileiro;

$4^{\circ}$ mapeamento de competências para inovar no enfrentamento das DTN; 
$5^{\circ}$ inovação através da abordagem HT para internalização dos fármacos \& medicamentos.

A interação promovida pelo Conselho à luz da HT estaria envolvendo os setores pertencentes do governo, universidades e empresas, como detalhado a seguir:

a) o governo federal promove para ele mesmo, através do MS a criação do CGC-DTN e este articula sua composição com demais atores, como apresentado na Figura 2;

b) o acompanhamento da evolução da RENAME configura importantes subsídios para as empresas produtoras de fármacos \& medicamentos, bem como para os LFO, no planejamento de ações de atualização e prospecção em seus portfólios;

c) para a internalização de fármacos \& medicamentos propriamente dita, é fundamental a sinergia e interação das empresas, institutos de pesquisa e governo. Para tanto, a disponibilização dos dados de mercado e de competências nacionais nestas áreas serviriam para munir os órgãos de fomento (Finep, CNPq etc.) na confecção de editais, formação de grupos de trabalho, cabendo ao Banco Nacional de Desenvolvimento Econômico e Social (BNDES) abrir linhas de financiamento, para as empresas organizarem seus planos estratégicos etc.;

d) o fortalecimento dos LFO e privados aconteceria através do fortalecimento nas políticas governamentais fomentadas pelas associações (ALANAC, ALFOB, Pró-genéricos) e executadas pelos governantes diante da consciência na defesa nacional em fármacos \& medicamentos para DTN;

e) o incentivo às competências nacionais e redes cooperativas é de extrema relevância para a valorização e independência brasileira na área de DTN, bem como indutor social para outros países menos desenvolvidos e que carecem sobremaneira nesta área, como a África. Não obstante, os países desenvolvidos são potenciais (através da imigração de pessoas contaminadas, casamentos etc.) em algumas DTN. Nestas ações, o MEC, MCT e os Institutos de Pesquisa disponibilizariam seus docentes e grupos de pesquisa, o INPI concederia informações estatísticas e resguardaria o país nas tecnologias desenvolvidas etc.;

f) concernente ao acesso às informações tecnológicas e medicamentos à população no Sistema Único de Saúde (SUS), a presença dos profissionais da ANVISA, MS, INPI e MEC seria fundamental para a socialização de suas bases de dados, bem como das ações e resultados obtidos pelo CGC-DTN;

g) a dinâmica do mercado para DTN deveria ser monitorada pelos Observatórios como, por exemplo, o OBTENDO - Observatório de Tendências da EQ/UFRJ e o Observatório da PI (Propriedade Intelectual) do INPI. Nesse sentido, a participação do Centro de Gestão e Estudos Estratégicos (CGEE), MDIC, MCT etc. é fundamental a fim de manter a retroalimentação de informações para as tomadas de decisões das associações, institutos, fundações e do próprio CGC-DTN.
A interação dos atores provenientes ora do Governo, universidade ou empresa sempre ocorreria em formas de comitês, ou "frentes de trabalho", a fim de acompanhar as ações e os resultados alcançados.

Nesse sentido, a atuação do CGC-DTN estará baseada no conceito da HT e dados desta tese, as "giradas" das hélices serão simultâneas e/ou dinâmicas entre si, afastando-se ou aproximando-se de acordo com a robustez ou fraqueza das conquistas. Essa interação seria constante até que se alcançasse o objetivo proposto no projeto inicial pelo Conselho.

Imaginando os movimentos impetrados por uma hélice de três pontas, podem-se desenvolver várias análises como, por exemplo:

- em um movimento “A” as organizações estão estáticas, atuando naquilo para o qual foram concebidas e exercendo sua competência total. Observa-se ainda que a interação seja distante, necessitando transpor um "vácuo" para se comunicarem. São totalmente formais (não há contato central da ponta das hélices - vértice);

- num movimento "B" aproximam-se das instituições, onde a troca de informações é facilitada pela retirada do "vácuo" que existia, porém há uma linha tênue, mas extremamente forte, para que nenhuma se intrometa nas atividades da outra (há contato somente na ponta central das hélices - vértice);

- já num movimento "C" parte de cada organização cede espaço e forma-se um campo de inovação comum a todas, onde esperam ganhar competência e lucrar com a nova atividade (há contato central das pontas das hélices - formam o vértice);

- para o "D" uma das entidades se afasta por algum motivo, desde questões internas a políticas ou financeiras, podendo retornar a qualquer momento (somente duas pontas da hélice continuam no vértice, a outra se afasta);

- o movimento "E" pode ser uma interação muito forte entre duas organizações, enquanto a terceira mantém a participação, mas sem a intensidade das demais (duas hélices estão quase que sobrepostas, enquanto a terceira continua somente com a ponta do vértice participando da operação);

- em um possível movimento "F" pode ocorrer uma cisão gerando uma nova organização, independente, mas com a devida competência, originada das três que a conceberam. Provavelmente, dentro do sistema inovativo em que a sinergia acontecia, se observou uma lacuna a ser preenchida no país e formou-se nova entidade, a qual passa a dar o suporte necessário ao projeto e começa "girar" nova HT num processo contínuo (retorna-se ao movimento "A", porém criou-se uma $4^{\mathrm{a}}$ hélice para um novo processo).

A ideia principal seria analisar os pontos fortes e fortalecer os fracos, com ideias novas ou de outros locais. No Quadro 2 encontramse, sinteticamente as novas características que deveriam permear as atitudes do CGC-DTN.

Nesse sentido, estabelecidas as responsabilidades, ações e parcerias estratégicas, se esperaria que o CGC-DTN:

Quadro 2. Características do CGC-DTN na visão hélice tríplice

\begin{tabular}{|lll|}
\hline Características & Renda e atividade empresarial total & Nova visão da hélice tríplice \\
\hline Motivação para empreender & Necessidade & Transformar necessidade em motivação \\
\hline Educação & $\begin{array}{l}\text { Grande concentração de empreendedores com } \\
\text { baixo nível educacional }\end{array}$ & Encontrar nichos para habilidades naturais \\
\hline Tecnologia e Inovação & Utilização de tecnologia antiga e baixa inovação & Utilizar tecnologia social \\
\hline Capitalistas de risco & Não se sentem atraídos por investir & Responsabilidade social \\
\hline Políticas de suporte ao empreendedorismo & $\begin{array}{l}\text { Formuladores não se mostram interessados em } \\
\text { dirigir programas para empreendedores de baixo } \\
\text { valor agregado }\end{array}$ & $\begin{array}{l}\text { Propor Políticas Públicas de geração de trabalho } \\
\text { e renda }\end{array}$ \\
\hline
\end{tabular}

Fonte: Abalizada na $5^{\text {a }}$ Triple Helix Conference - May, 2005 
- $\quad$ promovesse e atuasse em ações para a internalização de fármacos e/ou medicamentos para DTN;

- incentivasse a $\mathrm{P} \& \mathrm{D}$ de medicamentos, diagnósticos, acesso à informação e tratamento, bem como o fortalecimento da capacidade de pesquisa em regiões onde as DTN são endêmicas;

- fomentasse estudo da fase pré-clínica até sua colocação no mercado, seja com incentivos através de legislação, períodos de "graça" (incentivar os investimentos privados nacionais, discutir e criar dispositivo na legislação que favoreça a esses produtores um período de exclusividade no mercado, seja em impostos e/ou garantia de compra (poder de compra do Estado) etc.), voucher (proposta de mecanismo para ampliar o tempo de patente de algum medicamento de marca do detentor da mesma, a fim de compensar investimentos realizados em P, D \& I em DTN. Este dispositivo passaria por ampla discussão e estudo com entes governamentais) nas patentes, fundos setoriais etc.;

- promovesse estratégias como desafios no controle e à prevenção de DTN reemergentes e associadas a outras enfermidades, tais como a coinfecção HIV/tuberculose, doenças sexualmente transmissíveis, dengue, Chagas (vetor que transmite a doença misturado em alimentos - como a má limpeza durante a comercialização do açaí) etc.;

- definisse os passos críticos e tempos dos processos e movimentos das ações, contemplando as instruções, sinopses, líderes de grupos etc.;

- permitisse retorno periódico e reflexões que assegurem o aprendizado;

- executasse sempre os processos com transparência;

- clarificasse todos os procedimentos - clareza nas ações dos fatores críticos, escolhas estratégicas, a soberania nacional em torno do processo;

- atuasse sempre como política de Estado e não de Governo;

- conscientizasse os atores da oportunidade constante de aprendizado (inovação incremental e/ou radical);

- houvesse mapeamento constante do mercado de DTN.

As interações e sinergias entre todos os atores da HT para o CGC-DTN estão ilustradas na Figura 4.

Parafraseando Etzkowitz e Ranga, ${ }^{30}$ este modelo proporciona uma "transição sem fim" e uma "evolução contínua" para o combate às DTN, pois sempre existirá uma maneira mais apropriada para a realidade de cada local.

Com o CGC-DTN utilizando a inovação através das competências instaladas no $\mathrm{G}-\mathrm{U}-\mathrm{E}$, espera-se resultados práticos para a sociedade traduzidos em:

- investimento em P \& D para a indústria farmacêutica nacional;

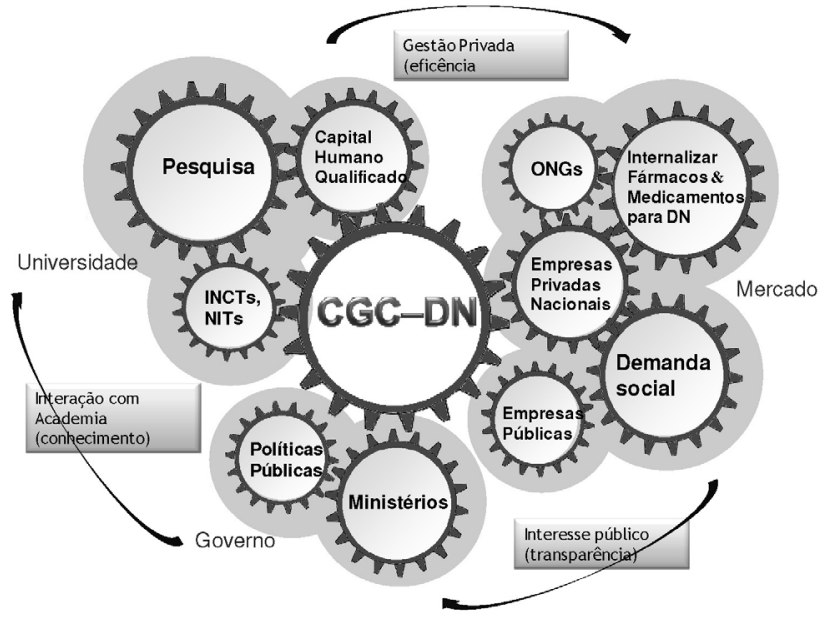

Figura 4. Interação dos atores no CGC-DTN. Elaborada utilizando conceitos de Etzkowitz. INCT - Instituto Nacional de Ciência e Tecnologia - criado através de fomento do CNPq; NIT - Núcleo de Inovação Tecnológica-

- consolidação de políticas favorecendo a produção farmoquímica interna e de medicamentos para DTN;

- fortalecimento dos LFO, com maior participação na produção de medicamentos para DTN elencados na RENAME;

- a universidade se torne empreendedora na socialização do conhecimento às empresas, através de sinergias e relação "ganhaganha";

- a Defesa Nacional e o CEIS sejam fortalecidos com redução nos custos para o sistema de saúde, reduzindo assim a dependência do exterior;

- diminuição do déficit existente na balança comercial em relação às importações de IFA e medicamentos para DTN;

- a população brasileira tenha melhoria no acesso às informações e tratamento para DTN;

- capacitação em DTN na P, D \& I.

No Quadro 3 observa-se a contribuição para a saúde pública brasileira com a consolidação geral da proposta, demonstrando a HT pela interação das competências existentes no G-U-E.

\section{CONSIDERAÇÕES FINAIS/CONCLUSÕES}

O Brasil possui competências estabelecidas no território nacional nas esferas do G-U-E. Os avanços alcançados em políticas na área de saúde são notórios, contudo, a sinergia para maximizar o tempo para

Quadro 3. Resumo das competências X lacunas nas doenças tropicais negligenciadas

\begin{tabular}{|lccc|}
\hline \multirow{2}{*}{ Lacuna } & \multicolumn{3}{c|}{ C o m p e t ê n c i a s } \\
\cline { 2 - 4 } & Governo & Universidade & Empresa* \\
\hline Pesquisa básica & MCT, MS, Fiocruz & MEC, ICT & ALANAC e ALFOB \\
Pesquisa pré-clínica & MCT, MS, Fiocruz, Institutos de Pesquisa & MEC, ICT & ALANAC e ALFOB \\
Fase clínica & MCT, MS, Fiocruz, Institutos de Pesquisa & MEC, ICT & ALANAC e ALFOB \\
Formulação & MCT, INPI, LFO, NIT & MEC, ICT & ALANAC e ALFOB \\
Registro & ANVISA & ALANAC e ALFOB \\
Produção & LFO & ALANAC e ALFOB \\
Acesso à população & MS, MJ, Senado, Câmara & Rede de distribuição \\
Farmacovigilância & MS, ANVISA & ALANAC e ALFOB \\
\hline
\end{tabular}

* Entende-se como privada ou pública ou ainda instituições - os Laboratórios farmacêuticos pertencentes à respectiva Associação, por exemplo: público (LFO), privados nacionais medicamentos (Biolab, Cristália etc.), privados nacionais fármacos (Microbiológica, Nortec etc.) 
fortalecer a defesa nacional no que tange às DTN se mostra ainda um grande desafio. Nesse sentido, as ações estratégicas intersetoriais são fundamentais para favorecer o acesso da população aos medicamentos e o uso racional dos mesmos, bem como as políticas no CEIS. Consequentemente, a superação dos desafios para o enfrentamento das DTN passa pela "afinação" de todos os atores (governo, universidades e empresas).

A PDP pode ser exemplo para desenvolvimento de tecnologia em medicamentos no país. Igualmente, parcerias com as universidades e instituições para testes clínicos devem fortalecer as competências locais para avançar na internalização de fármacos e medicamentos. Não obstante, deve-se fomentar as parcerias, de forma a verticalizar a fabricação de medicamentos para as DTN através de planejamento de produção estratégica para o país, utilizando as universidades na interação com P, D \& I incremental ou radical.

O potencial de capacidade instalada nos LFO deve ser fomentado na inclusão de programas voltados à internalização de tecnologias para a produção dos medicamentos para DTN, como parte integrante da cadeia do desenvolvimento tecnológico, onde é uma lacuna a ser vencida até a inovação mercadológica. Portanto, são fundamentais as sinergias de competências distintas para a verticalização do processo e desta forma conseguir um equilíbrio entre maximizar o sucesso e a sustentabilidade da inovação (incremental ou radical) de um produto para DTN.

A criação do Conselho, baseado nas interações propostas pelo modelo da HT, visa favorecer a internalização dos fármacos \& medicamentos em DTN através do acesso e capacidade de resposta dos produtores nacionais (público e privado), proporcionando ao sistema sustentabilidade, qualidade, equidade e eficiência. Portanto, o monitoramento das ações promovidas pelo CGC-DTN deve ser constante, de forma a validá-las ou redesenhá-las, para alcançar o objetivo principal de sua missão, que é internalizar os fármacos \& medicamentos para DTN.

Com a efetivação do CGC-DN, se esperaria criar um ambiente favorável à inovação no país, com ampliação da capacidade inovadora e expansão da base científica e tecnológica nacional para DTN. Não obstante, o desenvolvimento de uma base ampla de apoio e envolvimento da sociedade no enfrentamento desse agravo se transforma em elemento estratégico de política para o desenvolvimento nacional.

\section{MATERIAL SUPLEMENTAR}

Para melhor entendimento e visualização da proposta descrita neste artigo, foram construídas uma tabela e mais duas figuras, que se encontram disponíveis em http://quimicanova.sbq.org.br, na forma de arquivo pdf, com acesso livre.

A Tabela $1 \mathrm{~S}$ é relativa às atividades - ou sub-funções - ou sistemas de inovação, que foram descritas como tarefas por Markard e Truffer. A Figura 1S exemplifica a estrutura para o CGC-DTN segundo o conceito HT, através das etapas da gestão do conhecimento existente nos atores. A Figura $2 \mathrm{~S}$ demonstra a sinergia por meio de ilustração do "giro" da hélice tríplice nas ações do CGC-DTN.

\section{REFERÊNCIAS}

1. Estudos FeSBE I; As Especificidades do Sistema de Inovação do Setor Saúde: Uma Resenha da Literatura como Introdução a uma Discussão sobre o Caso Brasileiro, Universidade de São Paulo: São Paulo, 2000.

2. Fernandes, R. M.; Dissertação de Mestrado, Universidade Federal de Minas Gerais, Brasil, 2005.

3. Morel, C. M.; Acharya, T.; Broun, D.; Dangi, A.; Elias, C.; Ganguly, N.
K.; Gardner, C. A.; Gupta, R. K.; Haycock, J.; Heher, A. D.; Hotez, P. J.; Kettler, H. E.; Keusch, G. T.; Krattiger, A. F.; Kreutz, F. T.; Lall, S.; Lee, K.; Mahoney, R.; Martinez-Palomo, A.; Mashelkar, R. A.; Matlin, S. A.; Mzimba, M.; Oehler, J.; Ridley, R. G.; Senanayake, P. S.; Singer, P.; Yu, M.; Science 2005, 309, 401.

4. Buse, K.; Walt, G.; Bulletim World Health Organization 2000, 78, 549.

5. Morel, C. M.; Cad. Saúde Pública 2006, 22, 1522.

6. Vieira, K. P.; Albuquerque, E. M.; R. Econ. do Nordeste 2007, 3, 38.

7. Lastres, H. M. M.; Maciel, L. M.; Systems of Innovation and Development, Edward Elgar Publishers: Cheltenham, 2003.

8. Brasil, Ministério do Desenvolvimento, Indústria e Comércio Exterior, Instituto Euvaldo Lodi; O Futuro de Fármacos no Brasil: coletânea de artigos, IEL/NC: Brasília, 2004.

9. Brasil, Ministério da Saúde, Secretaria de Ciência e Tecnologia e Insumos Estratégicos; Produção Oficial de Medicamentos: diagnóstico, limitações e perspectivas, Gráfica Ministério da Saúde: Brasília, 2003.

10. Magalhães, J. L.; Tese de Doutorado, Universidade Federal do Rio de Janeiro, Brasil, 2010.

11. Buss, P. M.; Medicamentos no Brasil - inovação \& acesso, Editora Fiocruz: Rio de Janeiro, 2008.

12. Schumpeter, J.; Capitalismo, Socialismo e Democracia, Nova Cultural: São Paulo, 1985.

13. Gadelha, C. A. G.; Vacinas, Soros e Imunizações no Brasil, Editora Fiocruz: Rio de Janeiro, 2008.

14. Zaire, C. E. F.; Dissertação de Mestrado, Universidade do Estado do Rio de Janeiro, Brasil, 2008.

15. Forghieri, Y. H.; Psicologia fenomenológica: fundamentos, métodos e pesquisa, Editora Thomson Pioneira: São Paulo, 1993.

16. Humbert, M.; Globalisation and Glocalisation: problems for developing countries and policy (supranational, national and subnational) implications - Université Rennes I, Erudit: France, 2004.

17. Cruz, G. M.;Tese de Doutorado, Universidade Federal do Rio de Janeiro, Brasil, 2006.

18. Abdalla, M. M.; Calvosa, M. V. D.; Batista, L. G.; Rev. Cad. Adm. 2009, 2,3 .

19. http://www.thetriplehelix.org/, acessada em Janeiro 2011.

20. WHO - World Health Organization; Working to overcome the global impact of neglected tropical diseases - First WHO report on neglected tropical diseases, WHO Press: Geneva, October, 2010.

21. Antunes, A. M. de S.; Mangueira, A. C. S.; Quim. Nova 2005, 28 Suplemento, S112.

22. Antunes, A. M. S.; Magalhães, J. L.; Oportunidades em Medicamentos Genéricos - A Indústria Farmacêutica; $1^{\mathrm{a}}$ ed., Editora Interciência: Rio de Janeiro, 2008.

23. Bermudez, J. A. Z.; Cad. Saúde Pública 2007, 23, 744.

24. Etzkowitz, H.; Leydesdorff, L.; Research Policy 2000, 29, 109.

25. Etzkowitz, H.; Marina, R.; Hélice Tríplice. Universidade-IndústriaGoverno Inovação em Movimento, $1^{\mathrm{a}}$ ed., Editora PUCRS: Rio Grande do Sul, 2009.

26. Markard, J.; Truffer, B.; Research Policy 2008, 37, 596.

27. Magalhães, J. L.; Boechat, N.; Antunes, A. M. S.; RECIIS - R. Eletr. de Com. Inf. Inov. Saúde, (2011), doi:10.3395/reciis.v5i1.367pt.

28. Brasil, Ministério da Saúde; Resultados e Conquistas 2009 - uma prestação de contas à sociedade, Gráfica Ministério da Saúde: Brasília, 2009.

29. Brasil, Ministério da Saúde, SCTIE; Assistência Farmacêutica na Atenção Básica: instruções técnicas para sua organização, Gráfica Ministério da Saúde: Brasília, 2006.

30. Etzkowitz, H.; Ranga, M.; A Triple Helix System for Knowledge-based RegionalDevelopment: From "Spheres" to "Spaces", International Institute of Triple Helix, LaSalle Madrid: Madrid, 2010. 


\section{INTERNALIZAÇÃO DE FARMOQUÍMICOS \& MEDICAMENTOS PARA DOENÇAS TROPICAIS NEGLIGENCIADAS: PROPOSTA DE INTERAÇÃO ENTRE GOVERNO - UNIVERSIDADE - EMPRESA}

\section{Jorge Lima de Magalhães* e Núbia Boechat}

Departamento de Síntese em Fármacos, Área Gestão da Inovação Tecnológica, Fundação Oswaldo Cruz/Farmanguinhos, Rua Sizenando Nabuco, 100, 21041-250 Rio de Janeiro - RJ, Brasil

\section{Adelaide Maria de Souza Antunes}

Instituto Nacional de Propriedade Intelectual, Praça Mauá, 7, 20081-240 Rio de Janeiro - RJ / Escola de Química, Universidade Federal do Rio de Janeiro, 21949-900 Rio de Janeiro - RJ, Brasil

Tabela 1S. Relativo às atividades - ou sub-funções - ou sistemas de inovação (Tarefas descritas por Markard e Truffer). Traduzido da ref. 26

\begin{tabular}{|c|c|c|}
\hline Herkkert et al. (2007) & Bergek et al. (2005) & Chaminade e Edquist (2005) \\
\hline Atividades empresariais & Experimentação empresarial & Criando e evoluindo organizações \\
\hline Desenvolvimento e difusão do conhecimento & $\begin{array}{l}\text { Desenvolvimento e difusão do conheci- } \\
\text { mento }\end{array}$ & $\begin{array}{l}\text { Prover P \& D } \\
\text { Provisão de educação e treinamento }\end{array}$ \\
\hline $\begin{array}{l}\text { Orientação da pesquisa } \\
\text { Formação de mercado }\end{array}$ & $\begin{array}{l}\text { Influência na direção da pesquisa } \\
\text { Formação de mercado }\end{array}$ & $\begin{array}{l}\text { Articulação dos requisitos de qualidade vindos da demanda } \\
\text { Formação de mercado de novos produtos }\end{array}$ \\
\hline Mobilização de recursos & Mobilização de recursos & $\begin{array}{l}\text { Atividades de incubadoras } \\
\text { Financiamento dos processos de inovação } \\
\text { Provisão de serviços de consultoria }\end{array}$ \\
\hline Criação de legitimidade & $\begin{array}{l}\text { Legitimação } \\
\text { Desenvolvimento de externalidades posi- } \\
\text { tivas }\end{array}$ & $\begin{array}{l}\text { Criação/alteração de instituição } \\
\text { Redes e aprendizagem interativa }\end{array}$ \\
\hline
\end{tabular}

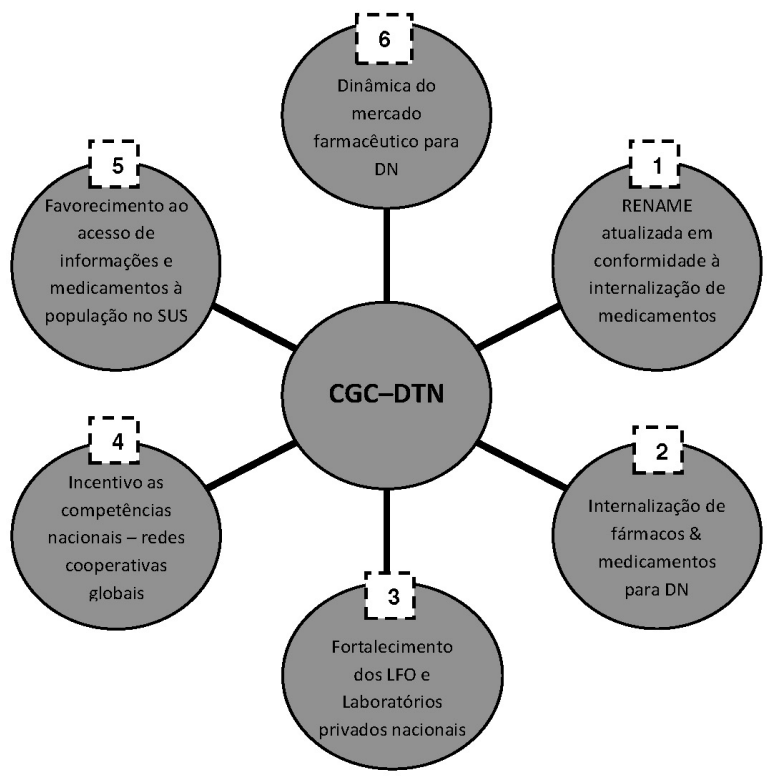

Figura 1S. Relativo à estrutura para o CGC-DTN segundo o conceito HT. Interações entre o G-U-E: (1) MS, ANVISA e Fiocruz; (2) Empresas (ALFOB, ALANAC, CNI, Incubadoras); (3) Governo, MS e Pró-genéricos; (4) MEC, MCT, Finep, MDIC, BNDES e INPI; (5) MS, ANVISA, INPI e MEC; (6) Observatório, MCT e CGEE

*e-mail: jorgelim@far.fiocruz.br
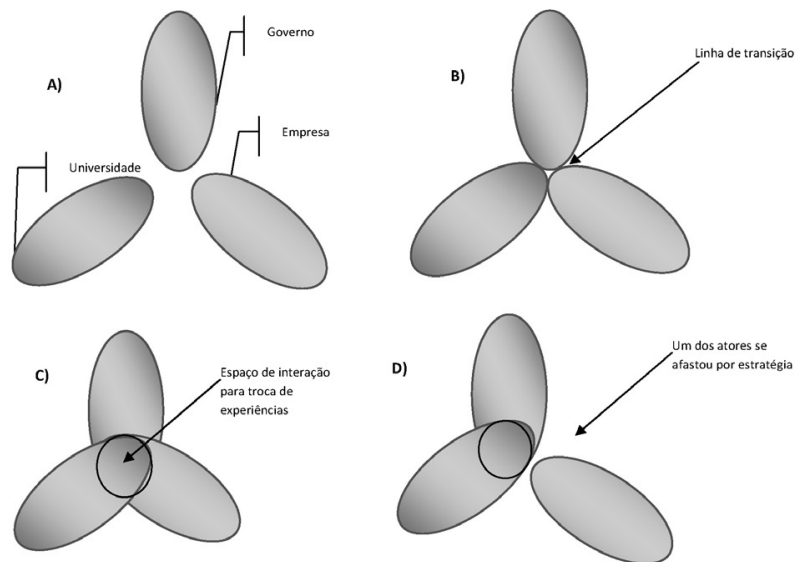

E)


F)

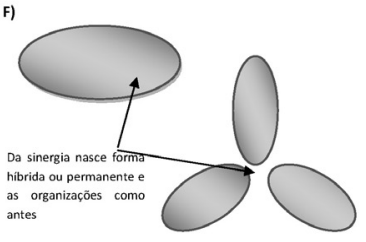

Figura 2S. Relativo à sinergia ilustrada no "giro" da hélice tríplice para as ações do CGC-DTN. Elaborada utilizando conceito de Etzkowitz, 2010 